NASA Technical Memorandum 100785 -

AIAA-88-0788

\title{
Numerical- Arc Segmentation Algorithm for a Radio Conference-A Software Tool for Communication Satellite Systems Planning
}

W.A. Whyte, A.O. Heyward, D.S. Ponchak, R.L. Spence, and J.E. Zuzek

Lewis Research Center

Cleveland, Ohio

(NASA-TM-100789) NOHERICAL ARC SEGHENTATION

N 88-22919

ALGORITHM FOR A BADIO CONPERENCE: A SOFT WARE

TOOL FOR COMUUNICATION SATELLITE SYSTEMS

PLANNING (NASA) $16 \mathrm{p}$ CSCI O9F

G3/17 0145967

Prepared for the

12th International Communication Satellite Systems Conference sponsored by the American Institute of Aeronautics and Astronautics Arlington, Virginia, March 13-17, 1988 
NUMERICAL ARC SEGMENTATION ALGORITHM FOR A RADIO CONFERENCE - A SOFTWARE TOOL FOR COMMUNICATION SATELLITE SYSTEMS PLANNING

W.A. Whyte, A.O. Heyward, D.S. Ponchak, R.L. Spence, and J.E. Zuzek

NASA Lewis Research Center

Cleveland, Ohio 44135

\section{Abstract}

The software package, Numerical Arc Segmentation Algorithm for a Radio Conference (NASARC), has been developed to provide a method of generating predetermined arc segments for use in the development of an allotment planning procedure to be carried out at the 1988 World Administrative Radio Conference (WARC - 88) on the Use of the Geostationary - Satellite Orbit and the Planning of Space Services Utilizing It. Through careful selection of the predetermined arc (PDA) for each administration, flexibility can be increased in terms of choice of system technical characteristics and specific orbit location while reducing the need for coordination among administrations. The NASARC software determines pairwise compatibility between all possible service areas at discrete arc locations. NASARC then exhaustively enumerates groups of administrations whose satellites can be closely located in orbit, and finds the arc segment over which each such compatible group exists. From the set of all possible compatible groupings, groups and their associated arc segments are selected using a heuristic procedure such that a PDA is identified for each administration. This paper discusses the various aspects of the NASARC (Version 2.0) concept and how the software accomplishes specific features of allotment planning.

\subsection{Introduction}

In 1985, the first of a two session "World Administrative Radio Conference on the Use of the Geostationary - Satellite Orbit and the Planning of Space Services Utilizing It" (WARC-85) was held in Geneva, Switzerland. Part of the overall objective of the Conference is to "guarantee in practice for all countries equitable access to the geostationary satellite orbit and the frequenc, bands allocated to space services". I WARC-88 is to accomplish this, in part, through the development of an allotment plan. At least one allotment in the plan is to be provided to each ITU member. Each allotment is to consist of:

- an orbital position within a predetermined $\operatorname{arc}$

- coverage to a specified service area for domestic services

- $800 \mathrm{MHz}$ bandwidth in the $\mathrm{C}$ and $\mathrm{Ku}$ band expansion frequencies given as:

4500 to $4800 \mathrm{MHz}$ and $300 \mathrm{MHz}$ to be selected in the band 6425 to $7075 \mathrm{MHz}$

and

10.70 to $10.95 \mathrm{GHz}, 11.20$ to $11.45 \mathrm{GHz}$, and 12.75 to $13.25 \mathrm{GHz}$.
In response to the decisions of WARC -85 regarding allotment planning for the Fixed Sateliite Service, the Numerical Arc Segmentation Algorithm for a Radio Conference (NASARC) software package has been developed for the purpose of identifying and selecting predetermined arcs (PDA's) for use by each administration in an allotment plan. An overview of the software and its related planning concepts is given in Reference 2.

The NASARC software first determines an exhaustive list of compatible groups of satellites providing coverage to corresponding service areas, and an arc segment over which each group may exist. Each compatible group consists of several service areas that are sufficiently separated geographically so that co-location or near co-location of their corresponding satellites will result in achieving a specified carrier-to-interference (C/I) ratio. The downlink single-entry carrier-to-interference ratios are calculated at an orbital separation equal to a user supplied grouping criterion for all possible pairs of systems at discrete arc locations. The calculated values are compared against a target $\mathrm{C} / \mathrm{I}$ to determine compatibility between service area satellites. Groups of compatible satellites are enumerated, where each member of a given group is compatible with every other member in the same group. The boundaries of the arc segment over which each compatible group may exist are identified.

Using a heuristic procedure, appropriate groups are then selected from a list of all compatible groups and their respective arc segments, and each is given a PDA within its corresponding arc segment. Members of the same group share a common PDA with all other members of the group. Given suitable input parameters, the final result of the NASARC process is a listing of the groups which contain all of the service areas represented in the input scenario and the boundaries of the unique predetermined arc segments associated with the members of those groups. Each service area may then be served from any one of a number of possible orbital positions within its PDA. Furthermore, because compatibility between allotted group members has been assessed by the NASARC software, minimal coordination is required between group members. Figure 1 illustrates by example three features of the NASARC concept for genorating predetermined arcs: PDA's may have different sizes, different allotted arc segments may have different numbers of satellites grouped within them, and the density of satellites per degree of orbit may vary.

The subsequent sections of this paper present a description of the features of the NASARC 
(Version 2.0) software package and detailed information about the function of each of the four NASARC program modules. Finally, the results of an example world scenario are presented and discussed.

\subsection{Features of the NASARC Sof tware}

The purpose of the NASARC software package is to generate a list of compatible groupings of service areas and associated arc segments and to select predetermined arcs for these service areas that will satisfy the requirements of an allotment plan. A service area may consist of the political/geographical boundaries of a single administration or the combined boundaries of a group of administrations which are geographically adjacent. Through careful selection of predetermined arcs, each administration will have flexibility in choosing satellite system technical parameters as well as flexibility in positioning a satellite within its predetermined arc limits. Additionally, the PDA's generated by the NASARC software package limit the need for coordination among administrations in a group due to the inherent compatibility within the shared predetermined arc.

The NASARC software package performs compatibility calculations for each pair of satellites/service areas under certain technical assumptions. The determination of compatibility assumes constant power flux density (PFD) at the edge of the minimum area ellipse covering each service area. Calculations are performed under clear sky conditions with homogeneous antenna patterns using a uniform single -entry carrierto-interference ratio. Certain bey features of the NASARC software package allow these baseline assumptions to be overridden, some on a service area by service area basis. An inhomogeneity factor may be used for each service area to override the constant power flux density assumption. Rain attenuation data can be included by utilizing a different inhomogeneity factor to override the clear sky assumption. Both satellite and earth terminal antennas can be individually specified for each service area to override the homogeneous antenna pattern assumption. The minimum ellipse assumption implies fairly simple satellite antenna technology. This assumption can be overridden either globally or on an individual service area basis by using fast roll-off satellite antennas to approximate shaped beam antennas.

These features also provide a means for handling two specific planning problems: existing systems and affiliated sets of service areas. Existing systems in the frequency bands being planned could be accommodated in at least two different ways. Such systems could be given the orbit location for which they had filed as an assignment with sufficient orbital guard zones to provide interference isolation; or, they could be given that specific orbit location within a PDA containing group members that are compatible with the existing system. Existing systems whose orbital locations are not fixed can be accommodated in the same manner as any other administration. In general, existing systems will have technical characteristics which may differ from the standardized technical parameters used in the planning process. To accommodate this, different antenna characteristics and inhomogeneity factors can be utilized for each existing system.

Another feature of the software allows administrations to illuminate several affiliated, but geographically separated, service areas from a single satellite location within the administration's predetermined arc. An affiliated set of service areas could consist of a single administration and its dispersed territories or could represent a subregional group of nonadjacent service areas. The affiliated set feature can also be used to simulate a multiple beam system as exemplified by one of the global coverage existing systems. This feature along with those previously mentioned will be described further in the following sections.

\subsection{System Inhomogeneity Factor}

The principal applications for the system inhomogeneity factor are overriding the constant power flux density assumption and representing existing systems. As previously described, NASARC assumes homogeneous systems and constant power flux density at the edge of the minimum area ellipse covering each service area. Although these assumptions are reasonable for planning purposes, it may be desirable to introduce some degree of inhomogeneity into the planning process. The primary reason that planning has commonly been performed with a homogeneous set of parameters is that it is difficult to assess the specific parameter values to associate with each satellite system before the intended purpose of the system has been identified. This is especially true of the varied systems in the Fixed Satellite Service. Different signal types may require different levels of interference protection. However, without specific knowledge of the levels required for each system, results obtained from randomly introduced inhomogeneities could be misleading. Hence, in general, it is desirable to conduct planning with the assumption of homogeneous systems.

Planners may wish to override the constant PFD assumption in order to investigate the effects of constant satellite transmitter power among satellites in a given scenario. The method of handling inhomogeneities which has been incorporated in the NASARC software involves modifying the threshold $\mathrm{C} / \mathrm{I}$ requirement (i.e., the homogeneous single-entry protection level) by inhomogeneity factors related to the wanted and interfering systems. The modified $\mathrm{C} / \mathrm{I}$ level becomes the target $C / I$ level used in the determination of pairwise compatibility in the NASARC software package.

The simulation of constant satellite transmitter power for all systems in a given scenario can be accomplished by calculating an inhomogeneity factor associated with each service area. This factor would represent the difference between the actual transmitter power in decibels (dB) and some user-defined reference power. For example, the reference power level could be the lowest satellite power level required by any satellite in a scenario. The inhomogeneity 
factor associated with this service area would be $0.0 \mathrm{~dB}$, while all other service areas would have inhomogeneity factors representing the difference between their required satellite power and this reference power level.

The inhomogeneity option can also be used to simulate the differences between an existing system's power or PFD level and the homogeneous values used by the other systems in the scenario. By utilizing the inhomogeneity factor and other NASARC features discussed herein, existing systems can be modeled in a realistic fashion for planning purposes.

\subsection{Rain Attenuation}

In addition to the general inhomogeneity factor, there is a specific inhomogeneity factor which can be used to take into account rain attenuation effects in determining pairwise compatibility. Use of this option overrides the clear sky assumption in the NASARC software package. Rain attenuation data for each service area at every test point for each discrete arc location is given in the precalculated ellipse data file. In a file input to NASARC, attenuation data is included for the expansion frequencies around both $C$ band and $K u$ band based on an annual percent outage rate of 0.01 using the CCIR rain model. Based on the frequency chosen for the scenario in question, the largest or worst-case attenuation value is chosen from all of the test points of a given service area at the appropriate arc location. This value is then adjusted to correspond to annual percent outage rates of 0.05 for C-band and 0.10 for Ku-band, which are the values suggested by the International Frequency Registration Board (IFRB). Although the attenuation values given in the ellipse data file can be quite large, the software limits the attenuation value to a maximum of $10 \mathrm{~dB}$ as used by the IFRB in their planning exercises. This limiting value can be changed if necessary. The attenuation value is then used as an inhomogeneity factor in the pairwise compatibility calculations.

The rain attenuation inhomogeneity factor may be used either in addition to the general inhomogeneity factor discussed in the previous section or by itself to examine the effects of rain attenuation on the clear sky analysis of homogeneous systems. Additionally, since this option can be utilized with or without the general inhomogeneity factors, several combinations of technical assumptions can be investigated to observe their varying effects on the predetermined arcs found by NASARC.

\subsection{Specification of Individual Antenna Parameters}

Another feature available in the NASARC software package is the optional specification of individual antenna parameters for each service area. This feature allows the specification of different earth station and/or satellite antenna types and parameters for any or all of the service areas for a given scenario. There are two types of earth station antenna patterns and nine types of satellite antenna patterns provided in NASARC (Version 2.0). The earth station patterns differ only in their sidelobe levels, one having a sidelobe envelope of $32-25 \log _{\varphi}$ and the other having a sidelobe envelope of $29-25 \log \varphi$, where $\varphi$ is the antenna of $f-a x$ is angle in degrees. In addition to the type of earth station antenna, both the earth station antenna diameter and efficiency can be specified for each service area.

Of the nine satellite antenna types available in NASARC, three are standard rolloff antennas, two are based on purely empirical formulas, and four are fast rolloff antennas. A fast rolloff antenna is one in which the gain from the edge of the minimum ared ellipse covering the service area declines at the same rate as that of an antenna having the minimum half-power beamwidth (HPBW). Such an antenna pattern can be used to approximate a shaped beam antenna where the individual beamlets are the same size as the minimum HPBW. The minimum HPBW is specified by the user as a global parameter for a given scenario. Some of the satellite antenna patterns require additional parameters which can be specified on an individual service area basis. Satellite antenna officiency may also be specified on an individual service area basis.

The individual antenna options are particularly useful in attempting to accurately model existing systems. These systems may have different earth station antenna diameters than those used for planning purposes which can be taken into account with the individually specified earth station antenna option. Additionally, the satellite antenna pattern of each existing s/stem can be approximated by choosing the pattern which most closely matches the actual pattern of the system in question.

\subsection{Affiliated Sets of Service Areas}

Some administrations may wish to illuminate several affiliated but geographically separated service areas from a single orbital location within that administration's PDA. Thus, this affiliated set of service areas would be defined by a number of non-adjacent service areas to be served from the same satellite. There are several possible applications for this option. For example, the affiliated set option could be used to accommodate a single administration and its dispersed territories. Similarly, the affiliated set option could be used for the case of nonadjacent administrations wishing to combine their requirements into a subregional grouping. Adjacent administrations in a subregional grouping would simply be regarded as a single combined service area which would be treated like any other service area in the NASARC process. Service areas within an affiliated set are assumed to be compatible with each other. In the aforementioned cases, the affiliated set is comprised of non-adjacent (i.e., geographically separated) service areas. Another potential use of the affiliated set option is to synthesize a multiple beam system. Each beam coverage area could be specified as a separate service area and then affiliated to examine the multiple beam interference effects with other service areas. Interference effects between beams are not examined 
because of their inherent affiliation with each other.

The NASARC software treats the members of any affiliated set as separate entities when determining pairwise compatibility, but does not determine the compatibility between the set members since this has been assumed to exist. The software then combines this information as if the affiliated set were indeed a single service area. Thus, an affiliated set is only considered compatible with another service area if each of its component members are individually compatible with the other service area in question and viceversa.

The affiliated set option along with the other software features mentioned in the previous sections allow for a large "ariety of planning scenarios to be investigated.

\subsection{Description of the NASARC Sof tware}

The purpose of the NASARC software package is to generate a list of compatible groupings of service areas and associated arc segments and to select predetermined arcs for these service areas that will satisfy the requirements of an allotment plan for a given set of technical parameters. Because NASARC enumerates all unique compatible groups at each arc location, the number of groups which are exhaustively enumerated for worldwide scenarios can become prohibitively large, thereby exceeding memory constraints of many computer systems. This is especially true for areas of the orbit which could potentially serve a large number of service areas (i.e., areas of the orbit where large numbers of service arcs overlap). These areas of "high density" are illustrated by the data given in Figure 2 for a typical set of service arcs in a worldwide scenario. Rather than processing the entire orbital arc all at once, the NASARC software operates on smaller segments of arc one at a time, thus covering the entire orbit in a piece by piece manner. The software enumerates groups within each segment, selects groups from this list and determines a predetermined arc for each group selected. By repeating the group enumeration and arc determination processes over successive segments of the orbital arc, an entire worldwide scenario can be processed.

This piecewise approach is utilized to alleviate the storage and execution time difficulties which result from the aforementioned high density areas of the orbital arc. Using an orbital density figure similar to the one given in Figure 2 , a segment of arc is selected where the relative density of service areas is low. Unique compatible groupings are enumerated for all service areas whose service arcs intersect the segment boundaries. From these groupings, suitable groups are selected and predetermined arcs are calculated within this segment. Once a service area has been given a predetermined arc, it is not considered in future segments. By progressively working from the areas of lowest density of the orbit to the areas of highest density, the number of service areas which need to be considered in the higher density areas is reduced accordingly.
Additionally, the piecewise approach described above preserves the integrity of the NASARC methodology for the development of predetermined arcs over the entire orbit as a whole. This is possible due to several important characteristics of the NASARC software package. First, the enumeration of compatible groupings occurs on an arc location by arc location basis with each arc location treated independently. Second, the arc determination process is done over the cumulative arc which includes past arc segments and allows for adjustment of PDA's over this entire are during each iteration of this process. Third, groups whose members have been given a PDA have their group arcs (i.e., the arc segment over which the group members are compatible) extended into future segments whenever possible, providing increased flexibility should repositioning become necessary. Finally, all groupings from past segments whose members have not been given PDA's but have the potential to be allotted an arc in a future segment are retained for future consideration. These characteristics enable the piecewise approach in NASARC to handle worldwide scenarios while utilizing less memory and achieving faster execution times than would be possible when attempting to process the entire orbital arc at once.

As illustrated in Figure 3 , the NASARC software package is comprised of four modules: NASARCO, the input program; NASARCl, the grouping program; NASARC2, the arc determination program; and NASARC3, the group arc extension program. The input program is run only once prior to the execution of the entire piecewise approach of NASARC. The two major purposes of the input program are to check certain input data files and to allow the user to input the various requiren technical parameters used throughout the NASARC software package. The remaining modules, NASARC1, NASARC2, and NASARC3, are executed successively in a repeated fashion for each segment of the total orbital arc. The grouping program determines pairwise compatibility between satellites and enumerates all unique compatible groupings at each arc location within a segment; it then determines the arcs over which these groupings exist. The arc determination program uses a heuristic process to select groups from the exhaustive list of unique groupings produced by NASARCl and calculates a predetermined arc for these groups. The group arc extension program extends the group arcs of the selected groupings into future segments whenever possible to allow for flexibility in adjusting the PDA's to get a successful plan. The following sections give a more detailed description of each of the NASARC program modules.

\subsection{NASARC 0 - The Input Program}

NASARC 0 is the first of the four NASARC modules to execute and it is only executed once throughout the piecewise approach. Its primary function is to interactively prompt the user for several key technical parameters for the desired scenario. These parameters include: the threshold single-entry downlink carrier-to-interference ratio, the downlink frequency, the satellite and earth station antenna specification codes, antenna efficiencies, and required antenna para- 
meters, the grouping criterion, and the minimum half-power beamwidth. The program also queries the user as to whether or not rain attenuation is to be considered. These parameters are stored in an input data file that is required by the remaining NASARC modules.

In addition to the primary function of accepting the prompted user inputs, NASARCD also checks various prestored input files for critical errors which would stop execution of the major NASARC modules. If any of the files are incorrectly structured, or certain parameter values are not within prescribed limits, the user is immediately notified of the error, which file contains the error, and at which point the error occurred. NASARCO then communicates to the remaining NASARC modules that an error is present in one of the input files by setting an error flag to yes, ' $y$ ', in the input data file. This allows the other NASARC modules to ascertain that a critical input error has occurred and to abort execution.

\subsection{NASARCl - The Grouping Program}

NASARCl enumerates compatible groupings of service areas and the arc segments over which each such grouping may exist, within the portion of the arc currently being examined. A compatible group of service areas consists of a set of service areas, each of which are compatible with all other :inbers on a pairwise basis.

Pairwise compatibility between service areas is assessed on the basis of the satellite sepa- ition reicured to allow systems serving both service areas to meet a target single-entry, downlink carrier-to-interference ratio (C/I). A user-supplied grouping criterion expresses (in degrees of orbital arc) the maximum orbital separation allowable between systems at which the target C/I value may be achieved for compatibility. That is, if systems serving two service areas achieve or exceed the target $C / I$ value at the grouping criterion spacing, they may be placed closely together in the orbit, and are hence compatible.

Over the range of orbital locations within the current segment, assessment of compatibility between all possible pairs of service areas takes place. Service arcs and segment boundaries are intersected to determine limitations on the feasible arc available to each service area in the current segment of the orbit. At each possible arc location within the current segment, compatibility is assessed for all possible pairs of service areas. Utilizing the results of the compatibility assessment, groups of systems that may be spaced closely together in orbit (i.e., those that are compatible) are enumerated at each discrete location in the current segment. These groups are periodically accumulated into a list of compatible groups, and arc segments over which each such group may exist, until all possibilities for compatibility in the current segment have been exhausted.

Prior to performing the operations described above, the NASARCl moduie collects input data such as service area and service arc data, ser- vice area affiliation data, technical parameter values, ellipse and polygon point data, and user specified program options - from a variety of input files. The NASARCl program then processes the input data to establish a scenario for the current segment.

The NASARCl module eliminates from consideration those service areas that have received PDA's in segments of the orbit that have been previously examined by the NASARC1, NASARC2, and NASARC3 modules. If a service area that has received a PDA is, in fact, an affiliated set of service areas, its member service areas are eliminated from the list of service areas to be considered within the current segment.

Service arcs of the remaining service areas are adjusted to conform to the boundaries of the current segment to be analyzed by NASARCl. If a service area's service arc does not intersect the current segment, the service area is eliminated from the list of service areas to be considered. A further check is performed, if needed, to determine if service arcs for all affiliated service areas within a set are present within the current segment. All members of an affiliated set of service areas must be able to be included in a compatible group, or no members will be included. Thus, if the service arc of any member service area of an affiliated set does not intersect the current segment, the remaining member service areas must be eliminated from the list of service areas to be considered. Elimination of service areas that need not be considered greatly reduces computer time required to analyze a given segment of the orbit.

When the list of service areas to be considered has been finalized, intersections of the modified service arcs are determined for all pairs of service areas. Next, for each service area, the NASARCl module determines the closest point, from a finite set of coordinates defining the service area, to the center of each of the other service areas. These points will represent the locations of the wanted area receivers in the compatibility assessment for pairs of service areas.

Beginning with the westmost possible arc location for any service area, the NASARCl module calculates a compatibility matrix and enumerates groups of compatible systems for that location. The location is then incremented and the process is repeated until all possible discrete arc locations within the current segment are exhausted. In construction of the compatibility matrix for each location, compatibility assessment for a pair of satellites is not performed unless the current location lies within the intersection of the feasible arcs for both service areas. Each entry of the compatibility matrix indicates whether or not a target $C / I$ value is achieved with a specified orbital separation (i.e., the grouping criterion) between two satellites, serving a pair of service areas, where the wanted satellite system is represented by the row index and the interfering satellite system is represented by the column index. Due to differences in the geographical extent of the service areas corresponding to the satellites (and thus, beam coverage size), the $\mathrm{C} / \mathrm{I}$ achieved when one satel- 
lite is seen as wanted and the other is regarded as an interferer may not be identical to the $\mathrm{C} / \mathrm{I}$ achieved when the roles of the two satellites are reversed. Thus, the compatibility matrix will initially be non-symmetric. Compatibility is indicated by a one (1); incompatibility is indicated by a zero (0)

Affiliated sets of service areas require special treatment in the determination of compatible groups of service areas. If two service areas are members of the same affiliated set, they will be automatically regarded as compatible. However, compatibility between each member service area and all other non-member service areas will be assessed in the manner described below.

The single-entry downlink $C / I$ value achieved by each pair of systems at the orbital separation specified by the grouping criterion is calculated from antenna discrimination achieved on the downlink. The characteristics required for this calculation are those representing the satellite transmit antenna pattern, and the earth station receive antenna pattern. The achieved $C / I$ value is calculated at a spacing between satellites that is equal to the grouping criterion. In NASARC, achieved C/I is represented by the total downlink antenna discrimination. Using the interfering satellite's antenna pattern and the wanted service area's earth station antenna pattern, the total downlink discrimination is determined as shown in figure 4. The two service areas are considered to be compatible if the achieved $C / I$ value meets or exceeds the target C/I. Service areas are found to be incompatible if the achieved $\mathrm{C} / \mathrm{I}$ is less than the target $\mathrm{C} / \mathrm{I}$ or if the arcs of the service areas in question do not intersect. In the latter case the calculations described above are not performed.

When evaluation of the achieved $c / I$ values has been completed at a location for all pairs of service areas, the compatibility matrix for the location is made symmetric. Each entry of the compatibility matrix corresponding to a row and column index is compared to its corresponding entry - that with row and column indices transposed. If both entries indicate compatibility, ones are present in the corresponding locations of the compatibility matrix. If either or both of the matrix entries contains a zero, zeros are placed in both corresponding locations of the matrix. Diagonal entries will be set to one, as each service area is regarded as being compatible with itself.

The compatibility matrix is now symmetric; symmetry is required for the enumeration of groups of compatible service areas at the current location. A compatible group will consist of a set of service areas whose members are each compatible with all other members. Affiliated sets of service areas will, once again, be treated in all-or-nothing fashion; rows and columns of the compatibility matrix that correspond to single service areas that are members of an affiliated set must be consolidated into a single row and column that expresses the set's compatibilities with other, non-member service areas.
The symmetric compatibility matrix may be regarded as a collection of vectors - one vector for each service area - which expresses compatibility between that service area and all others. To enumerate groups of compatible service areas, each vector is examined in turn. Each vector will have a one in the element corresponding to its own service area index, and additional ones and zeros indicating compatibilities with other service areas. By exhaustively examining all possible compatibilities, a complete list of compatible groupings for the current arc location is found. When a large number of such groups have been determined, the groups and arc locations are accumulated into a list of groupings and the arc segments over which they have been found to exist. When all locations within the current segment have been exhausted, the final list of groups and group arcs is output by NASARC l to a file that is utilized as input by the NASARC2 module.

\subsection{NASARC2 - The Arc Determination Program}

The primary function of NASARC2 within the NASARC software package is to select appropriate groupings from among those enumerated by the NASARCl program within the current segment boundaries, and to identify predetermined arcs associated with the groupings selected. The output of NASARC2 is stored in an intermediate file which contains a listing of selected groupings and their predetermined arc boundaries. After all seyments have been examined using the NASARC piecewise approach, the NASARC2 output file forms the basis of results for the NASARC report file generated by NASARC3. The details of the grouping selection process, and the PDA generation are contained in the following discussion.

One of the first functions performed by the NASARC2 software is the generation of service area information from the output of NASARCl. The service area information is used to generate figures of merit (FOM's) related to each service area and its groupings. This information consists of a compilation of the number of groups containing each service area and the service arc for each service area represented in the current segment. These parameters seem fairly simple in concept, but are very important in critical decision making areas, such as, determining which service area should be accommodated first and which of its groupings is to be selected. The process of updating the service area information is carried out each time a grouping is given an allotted arc because it is no longer necessary to consider members within the allotted groups.

With the incorporation of the piecewise approach into the NASARC methodology, it becomes necessary to prioritize the service areas for accommodation within each segment. The prioritization is done to ensure that the service areas having the most limited allotment opportunities may be accommodated first. Each service area receives a priority designation according to its remaining service arc outside of the current cumulative arc. The cumulative arc encompasses the current arc segment being processed and all previously examined segments. Service areas 
whose service arcs are entirely encompassed by the cumulative arc are given the highest priority, Pl, meaning that they must be accommodated within the current segment. The second highest priority level, P2, is given to service areas whose remaining service arc, outside of the cumulative arc is 20 degrees or less. Finally, a priority, P3, is given to service areas whose remaining service arc exceeds 20 degrees.

Once the service area information has been generated and prioritization has taken place, NASARC2 is ready to begin the selection of groups and generation of PDA's. The first step in the approach is to select a critical service area from the list of unaccommodated service areas. The decision as to which service area to select is made based upon two figure-of-merit factors (FOMO and FOMI). FOMO relates to the priority of the service area based on remaining service arc outside of the current cumulative arc. FOMI sorts the service areas within each of the priority levels by the number of groupings in which each service area appears. Based on FOMO and FOM1, the service area appearing in the fewest number of groups, from among those service areas with the highest priority ( $P 1$ followed by $P 2$ followed by $P 3$ ) is selected as the critical service area. This method provides a high probability of accommodating remaining service areas in multi-member groups, since the critical service area affects the least number of remaining groups. Additionally, this approach addresses the most difficult service areas to accommodate first, thereby reducing the possibility for unallotted service areas.

Following the seiection of the critical service area, the next step is to select a groupins that contains the critical service area (i.e. the critical grouping). The grouping selection process also employs two figure-of-merit factors (FOM2 and FOM3) simultaneously. FOM2 is a measure of the number of members in each grouping which contains the critical service area. FOM3 is a measure of the minimum group arc that must be available for a given grouping in order that an arc can be allotted to the grouping.

The determination of FOM 3 , the length of the allotted arc for a given group, is based on the following formula:

$A L=K 1 *(N-1) * G R P+K 2 * T(i)+K 3 * N+K 4$ eq. 1 where:

$$
\begin{aligned}
A L & =\text { Allotted arc length (degrees) } \\
N & =\text { Number of grouping members } \\
G R P & =\text { Grouping criterion (degrees) } \\
T(i) & =\text { Transitional arc length, related } \\
& \text { to grouping, } i \text { (degrees) }
\end{aligned}
$$

$K 1, K 2, K 3, K 4=$ User specified constants

All of the entries in the allotted arc length equation, with the exception of $N$, the number of members in a given group, are directly controlled by the user. Of the four constants, K1, K2, K3 and $\mathrm{K} 4$, at least one must be nonzero in order to avoid the trivial solution of $A L=0$. Each of these constants has a different effect on the calculation of $A L$ for a given grouping. $K I$ affects the emphasis of the grouping criterion on the allotted arc length. $K 2$ affects the emphas is of the transitional arc length related to each grouping on the allotted arc length. K3 allows the user to base the allotted arc length on the number of members the group contains while $K 4$ allows the user to increase or decrease the size of all allotted arcs by a constant value. Because the NASARC software performs operations at discrete arc locations, the value calculated for the allotted arc length is rounded up to the nearest integer degree.

The allotted arc length equation is the basis of figure-of-merit factor, FOM3. If a grouping containing the critical service area has a group arc greater than or equal to the necessary allotted arc length, it is retained for consideration as the critical grouping. The groupings that are retained are then judged on the basis of FOM2 in order to select the critical grouping. The grouping having the highest FOM2, or largest group-size is then selected as the critical grouping.

At this point, NASARC2 has selected a critical service area and a corresponding critical grouping containing the service area. NASARC2 must now check if any priority P1 service areas are missing from the NASARCl output file before it can begin allotting shared PDA's. If any $P 1$ administrations are missing, they are given individual allotted arcs within their service arcs. Once this is done, the next major task is to determine where in the geostationary orbit within the group arc constraint, the grouping's allotted arc should be located. The locations of past allotted arcs are subject to movement within their respective group arcs as additional groupings are selected to receive allotted arcs.

The objective of the allotted arc procedure is to determine an allotted arc for the critical grouping of length, $A L$, within its group arc, while maintaining allotted arcs for all previous selected groupings. Three different heuristic approaches of varying complexity are utilized in the software to achieve the objective. The first approach is referred to as the "zero density" allotted arc routine and is the simplest in concept: find a vacancy in the geostationary orbit that can accomodate the grouping's required allotted arc length within its group arc constraint.

The second and third approaches are referred to as the "push" and "shuffle" allotted are routines and involve a greater degree of complexity in determining allotted arcs than the zero density routine. Both routines involve clearing an orbital arc width of the required length for the critical grouping by relocating existing allotted arcs; exactly how this is done depends on the routine. These two routines are accessed, if and only if, the zero density routine is unsuccessful in finding an allotted arc for the critical grouping.

The objective of the push allotted arc routine is to clear the arc space required by an entering allotted arc for a critical grouping by pushing intersecting existing allotted arcs out and beyond the entering arc boundaries. The 
approach employed is basically as follows: the entering allotted arc is searched for intersections with existing allotted arcs. At this point, the program attempts to push the existing allotted arcs westward out of the entering allotted arc. If the existing allotted arcs that were pushed now overlap other existing allotted arcs, they too are pushed in order creating a chain reaction. This push attempt is completed when either an existing allotted arc becomes constrained by its group arc boundary, in which case the push was unsuccessful; or no overlap exists, and the push is considered successful. If the westward push is unsuccessful, the existing allotted arcs that remain intersecting the entering allotted arc are pushed eastward. If the eastward push is successful, the routine is exited with a new solution. If it is unsuccessful the shuffle routine is accessed.

The basic objective of the shuffle allotted arc routine is principally the same as that of the push routine: clear the arc space required for a given entering allotted arc for a critical grouping, by relocating any existing allotted arcs that intersect the entering arc boundaries. However, the approach taken to achieve this objective differs. In the shuffle routine, existing allotted arcs that intersect the entering arc boundaries are relocated to zero density, or vacant regions outside the entering allotted arc within their respective group arc constraints. In view of the fact that only existing allotted arcs that directly intersect the entering arc boundaries are moved (the rest remaining fixed) this approach is less complicated than the push routine Also, because less group arc constraints are involved, it can handle cases where the push routine would be unsuccessful in finding a solution.

Accessing the push and shuffle routines is done consecutively for each entering allotted arc. If either routine achieves a solution, updated allotted arcs are returned to the main program. If both routines exhaust the list of potential allotted arcs for a given critical grouping without finding a solution, a new grouping containing the critical service area is sought. If the critical grouping had been a single priority $P 1$ service area, it is flagged for special handling and a new critical service area is selected. The combination of the three heuristic approaches (zero density, push, and shuffle) offers a high probability of successfully formulating predetermined arcs for all service areas given a suitable set of technical parameters.

A NASARC2 run is completed when either of two conditions occurs. The first is when all service areas have been either given a PDA or flagged for special handing. The second cause of termination is the result of the incorporation of the NASARC piecewise approach. When the arc space within the current segment is exhausted, NASARC2 halts execution and stores the unallotted groups in a special file for use in the next segment. The intermediate results are also examined by NASARC 3 to determine if their group arcs can be extended into the next segment to increase the amount of repositioning flexibility in the arc determination procedure.

\subsection{NASARC3 - The Group ArC Extension Program}

NASARC 3 examines compatible groups of service areas that have received allotted arcs through the arc determination process (the NASARC2 module), in order to determine if their group arcs may be extended into the next segment to be analyzed by NASARCl and NASARC2. This operation is performed in order to retain as much flexibility as possible in the arc determination process as applied to all segments of the orbit; that is, if adjustment of existing allotted arcs is necessary in order to accomodate entering allotted arcs, the full group arc will be available for the adjustment.

As discussed in the previous section, when a group of compatible systems has been selected by NASARC2 to receive an allotted arc, the NASARC2 module will examine the arc segment over which the group has been found to exist and will select a suitable portion of the group arc to become the group's allotted arc. It may become necessary to relocate existing allotted arcs, by moving them from their original locations, in order to accommodate entering allotted arcs. However, a relocated allotted arc for a compatible group of service areas must still lie within that group's group arc. For this reason, the full extent of each group arc into the next segment to be andlyzed must be determined.

Group arc extension is accomplished by the NASARC3 module in much the same way that grous arCs are determined by the NASARCl module; however, the computational effort required is considerably less. In NASARCl, all possible compatible groups of service areas, and their group arcs, are found within the segment currently being examined; in NASARC3, the group arc that exists for known, previously selected groups of service areas is determined within the next segment to be analyzed. Thus, analysis of compatibility is restricted to a small, known set of service areas, and exhaustive enumeration of compatible groupings need not take place.

In order to determine both the direction of, and limits upon, the group arc extension to be performed, NASARC3 obtains the next segment to be analyzed from the appropriate input file. If no segment of the orbit remains to be examined, NASARC3 produces the NASARC report file containing all results of the NASARC modules for all segments of the orbit that the user has chosen to analyze.

If a segment of the orbit remains to be examined, a group of compatible service areas and its corresponding group arc is retrieved from the intermediate file produced by the NASARC2 program. The group arc limits are compared with the eastern and western longitudinal limits of the next segment to be examined, in order to determine if group arc extension is possible. If the eastern limit of the group arc adjoins the western limit of the next segment, group arc extension is a possibility and the extension will be performed in the west-to-east direction. If the western limit of the group arc adjoins the eastern limit of the next segment, yroup arc extension is possible in the east-to-west direction. If the grc sp arc does not adjoin the next 
segment to be considered, the NASARC3 module returns to the intermediate file to obtain and check the next group and group arc. When all groups are exhausted, the intermediate file is updated with the extended group arcs and the program terminates.

If the group arc for the current group of service areas adjoins the next segment of the orbit to be processed, the NASARC3 module will extract information similar to that required for NASARCl from various input files. The service arc of each service area in the current group must intersect the arc segment to be examined for group arc extension; if a service area's service arc does not intersect the arc segment, the group arc may not be extended. If this is the case, the next group and group arc are selected from the intermediate file.

The segment of the orbit into which the group arc will be extended provides both the longitudinal limit of extension and the direction in which the group arc may be extended. The initial arc location is set to the appropriate western or eastern longitudinal limit of this segment; this arc location will be incremented if the group arc may be extended in the west-to-east direction, or decremented if extension is in the east-to-west direction.

Over the segment of arc to be considered, pairwise compatibility between service areas is assessed for each pair of service areas within the current group whose arc is to be extended, as in NASARCl. At each discrete arc location where compatibility between all service areas within the group still holds, the appropriate group arc boundary is adjusted to encompass the new location. When the group of service areas no longer continues to be compatible, or all locations within the segment have been examined, the next compatible group is retrieved from the intermediate file and the process is repeated.

When all groups and group arcs have been examined for extension into the next segment, the intermediate file will be updated to reflect the revised group arcs. When this operation has been performed, the NASARC 3 module concludes operation.

\subsection{World Scenario Example}

The NASARC software was applied to a worldwide scenario to demonstrate the process by which predetermined arcs (POAs) are generated. The scenario included 208 service areas identified by the IFRB. No existing systems were considered. of the 208 service areas, 175 service areas represent individual administrations, and the remaining 33 service areas represent 7 affiliated sets ranging in size from two to twelve members.

Table 1 presents the input parameters to the NASARC modules for this example. The NASARC POA generation was based on achieving a $32.0 \mathrm{~dB}$ single-entry downlink $\mathrm{C} / \mathrm{I}$ for systems operating in the Ku band expansion frequencies. The grouping criterion was specified as $0.5^{\circ}$ indicating that pairs of satellites in the same group meet the required $\mathrm{C} / \mathrm{I}$ at an orbital separation of $0.5^{\circ}$ or less. All service areas were set to operate with the default antenna parameters, of which the earth station antenna was specified to have a sidelobe envelope of $32-25 \log \varphi$ and the spacecraft antenna was specified to be the standard roll-off satellite antenna pattern resulting from the 1983 Regional Administrative Radio Conference (RARC '83). Within the scenario arcs file, wherever possible, all service areas were given a service arc corresponding to a $10^{\circ}$ elevation angle. Also, the effect of rain fade on the downlink was examined.

Table 1 also gives the constants used to specify the allotted arc equation. For this example, $K l=2, K 2=0.8$, and $K 3=K 4=0$. These values selected for the constants indicate that each grouping selected by the NASARC2 module would receive an allotted arc of length twice the amount required to accomodate the group members plus eighty percent of the calculated transitional arc length.

Table 2 presents the east and west boundaries of the ten orbital arc segments used to process the worldwide scenario. By comparing these segments to Figure 2 , it can be seen that the NASARC software was setup to begin in the least dense area (the Pacific ocean region), and by progressively reducing the peak was able to process the highest density area above ITU Region $l$ in the last segment. Using this approach, the entire example was completed in $20.2 \mathrm{CPU}$ minutes on an Amdahl 5860 mainframe computer having an instruction rate of 13 Mips.

The NASARC final results of the world scenario example are presented in Table 3 and illustrated in Figure 1 Three character codes are used to represent individual service areas or affiliated sets of service areas in the NASARC results. Wherever possible, the standard ITL three letter country codes are used. A graphical presentation of the selected groupings and their corresponding allotted arc boundaries is given in Figure 5. The final results show that the NASARC software was able to provide a solution that would accomodate all 208 service areas within $236^{\circ}$ of orbital arc and meet the technical requirements of the plan without producing any single administration allotments.

A total of 54 groupings containing the 208 service areas were selected by the NASARC software. The grouping size ranged from two members to eight members with a mean size of approximately four. Figure 5 shows the placement of allotted arcs for these gro:pings within the geostationary orbit (GSO). Unused areas of the orbit totalling $124^{\circ}$ could be utilized in subsequent iterations of the software to increase the size of the allotted arc for each grouping by changing the allotted arc length constants (eq. 1). This would result in greater flexibility in the choice of system technical parameters or in orbital positioning. Similarly, the portions of unused orbit could be utilized in incorporating larger scenarios which include existing systems.

\section{$\underline{5.0 \text { Conclusions }}$}

This paper has presented a detailed discussion of the NASARC software package for 
communication satellite systems planning. The purrose of this software tool is to provide a medls of satistying the iequirements of the allotment plan, to "... permit each administration to satisfy requirements for national services from at least one orbital position within a predetermined arc." This objective is accomplished through the exhaustive enumeration of compatible groupings of service areas, the selection of particular groupings and the identification of associated predetermined arcs. The NASARC concept assesses compatibility between systems over the entire PDA at the time of plan generation, thus allowing administrations to satisfy their requirements from any one of a number of possible orbital positions within a predetermined arc. The selection process is a heuristic procedure that utilizes several figure of merit factors designed to accommodate the most difficult allotment problems first. The task is to select groupings and generate predetermined arc sizes such that the requirements of all administrations are met before the available arc is exhausted. As with any approach to development of an allotment plan, the ultimate success of the approach will depend upon the selected values of technical parameters.

TAELE 1 - NASARC INPUT PARAMETERS

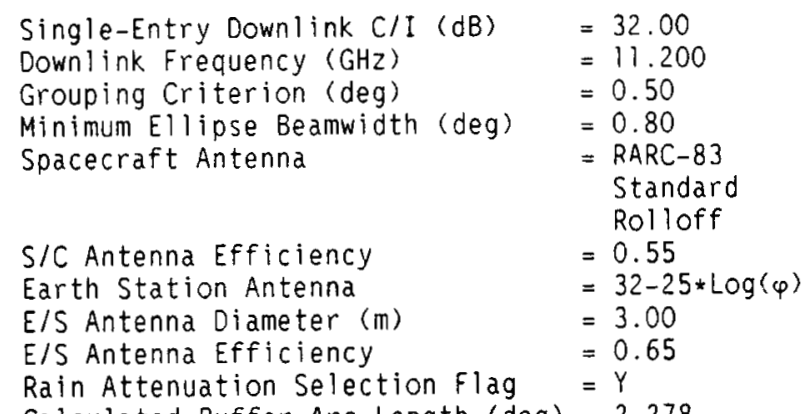

Calculated Buffer Arc Length (deg) $=2.278$

Constants for Allotted Arc Length

$$
\begin{aligned}
& K 1=2.00 \\
& K 2=0.80 \\
& K 3=0.00 \\
& K 4=0.00
\end{aligned}
$$

An example has been presented which demonstrates application of the NASARC concept to a worldwide scenario. The requirements of all systems were fully accommodated while allowing freedom of choice in the positioning of satellites and flexibility in the selection of system technical parameters.

\section{References}

l"Final Acts of the World Administrative Radio Conference on the Use of the GeostationarySatellite Orbit and the planning of space Services Utilizing It," First Session, Geneva, 1985.

2"An Allotment Planning Concept and Related Computer Software for Planning the Fixed Satellite Service at the 1988 Space WARC." Miller, et al, prepared for the Global Telecommunications conference, Sponsored by the Institute of Electrical and Electronics Engineers. Tokyo, Japan, November 15-18, 1987.

TABLE 2 - NASARC SEGMENT INPUT DATA

(Degrees East Longitude)

$\begin{array}{crr}\text { Segment } & \text { Western Boundary } & \text { Eastern Boundary } \\ 1 & 164.0 & -160.0 \\ 2 & 104.0 & 163.0 \\ 3 & 85.0 & 103.0 \\ 4 & -159.0 & -106.0 \\ 5 & -105.0 & -58.0 \\ 6 & 72.0 & 84.0 \\ 7 & -57.0 & -42.0 \\ 8 & -41.0 & -20.0 \\ 9 & -19.0 & 0.0 \\ 10 & 1.0 & 71.0\end{array}$

\section{ORIGINAL PAGE IS DE POOR QUALITY}


TABLE 3 - NASARC FINAL RFSULTS

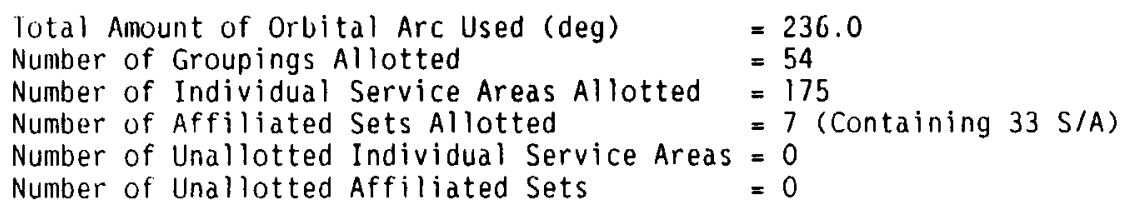

Group Members

CKH PHL SLM

DBØ FJI NZ2 PIC TON

MEX PRU

ABW GTM

CTR DMA

BAH NCG TRO

CA2 CUB PNR PRG SLV SUR VCT

BOL IJSA

CAN CLM

BL 2 EQA VEN

GNB SCN

ARG BRB HND SRL

CPV DOM GUY LBR

ATN HTI MTN

CHL. GMB GRO JMC POR TGO

ATG MLI URG

MRC STP

ALG ISL

OOO ON2

GNE HOL

$G A B$ IOO

$B \emptyset \emptyset$ BDI BEL MLT SMR

$\triangle C \emptyset$ BUL ISR ZMB

AFS QFØ LBY SEN

ALB DOR

SYR TCH

BHR COG CTI CYP OGO KFN

MWI POL QAT

EGY GHA SUI

AFG FNL YUG

GRC IRN LUX

DOO NIG TUN

BFA CAF JOR LIE

MCO NOR PAK ROU

AUT BEN

GUI OMA SØO TUR

HNG IRL LBN NGR TZA

CVA $\oslash \mathrm{F} \emptyset$ ZAI

CME NMB URS

AGL ETH IRQ

BOT IND SDN

DJI MOZ TCD

CHN COM RRW SWZ

ARS LSO

THA UGA UR2 YEM ZWE

SOM VTN

INS MOG

KRE KWT NPL YMS

OAØ BGD MAU MLA MNG SEY UAE

$B R M$ MLD

CLN LAO NZL UR3

CBG CK2 KOR SNG VUT

BRU PNG

AUS JØØ KIR NIU NRU OCE TKL TUV

\section{Allotted Arc Boundaries}

$-176.0 \quad-172.0$

$-172.0 \quad-166.0$

$-139.0 \quad-136.0$

$-136.0$

$-132.0$

$-128.0$

$-124.0$

$-116.0$

$-113.0$

$-110.0$

$-86.0$

$-83.0$

$-78.0$

$-73.0$

$-69.0$

$-62.0$

$-56.0$

$-52.0$

$-49.0$

$-46.0$

$-40.0$

$-37.0$

$-31.0$

$-26.0$

$-18.0$

$-15.0$

$-12.0$

$-5.0$

1.0

8.0

12.0

16.0

20.0

25.0

30.0

35.0

40.0

46.0

67.0

72.0

76.0

80.0

85.0

90.0

94.0

100.0

104.0

107.0

112.0

135.0

141.0

164.0

171.0

174.0
$-133.0$

$-129.0$

$-124.0$

$-116.0$

$-113.0$

$-110.0$

$-106.0$

$-83.0$

$-78.0$

$-73.0$

$-69.0$

$-62.0$

$-58.0$

$-53.0$

$-49.0$

$-46.0$

$-43.0$

$-37.0$

$-31.0$

$-26.0$

$-21.0$

$-15.0$

$-12.0$

$-5.0$

$-1.0$

5.0

12.0

16.0

20.0

25.0

30.0

33.0

40.0

46.0

50.0

71.0

76.0

80.0

84.0

90.0

93.0

100.0

103.0

107.0

112.0

120.0

138.0

146.0

170.0

174.0

$-177.0$
Group Arc Boundaries

$173.0 \quad-161.0$

$-139.0 \quad-106.0$

$-138.0 \quad-22.0$

$-132.0 \quad-16.0$

$-128.0 \quad-17.0$

$-124.0-106.0$

$-119.0 \quad-110.0$

$-113.0 \quad-52.0$

$-124.0 \quad-106.0$

$-86.0 \quad-72.0$

$-83.0 \quad-72.0$

$-78.0 \quad-59.0$

$-73.0 \quad-63.0$

$-69.0 \quad-58.0$

$-66.0 \quad-58.0$

$-56.0 \quad-43.0$

$-52.0 \quad-46.0$

$-51.0 \quad-46.0$

$-50.0 \quad-41.0$

$-40.0 \quad-27.0$

$-37.0 \quad-20.0$

$-33.0 \quad-21.0$

$-28.0 \quad-21.0$

$-18.0 \quad-1.0$

$-16.0 \quad-1.0$

$\begin{array}{ll}-13.0 & 0.0\end{array}$

$-16.0 \quad-1.0$

$1.0 \quad 45.0$

$8.0 \quad 46.0$

$9.0 \quad 18.0$

$12.0 \quad 52.0$

$12.0 \quad 32.0$

$25.0 \quad 33.0$

$29.0 \quad 70.0$

$35.0 \quad 47.0$

$12.0 \quad 46.0$

$45.0 \quad 61.0$

$67.0 \quad 71.0$

$72.0 \quad 82.0$

$72.0 \quad 80.0$

$72.0 \quad 84.0$

$74.0 \quad 96.0$

$86.0 \quad 95.0$

$85.0 \quad 100.0$

$39.0 \quad 103.0$

$104.0 \quad 111.0$

$105.0 \quad 113.0$

$108.0 \quad 120.0$

$121.0 \quad 138.0$

$136.0 \quad 146.0$

$156.0 \quad 172.0$

$171.0 \quad 174.0$

$168.0-177.0$ 


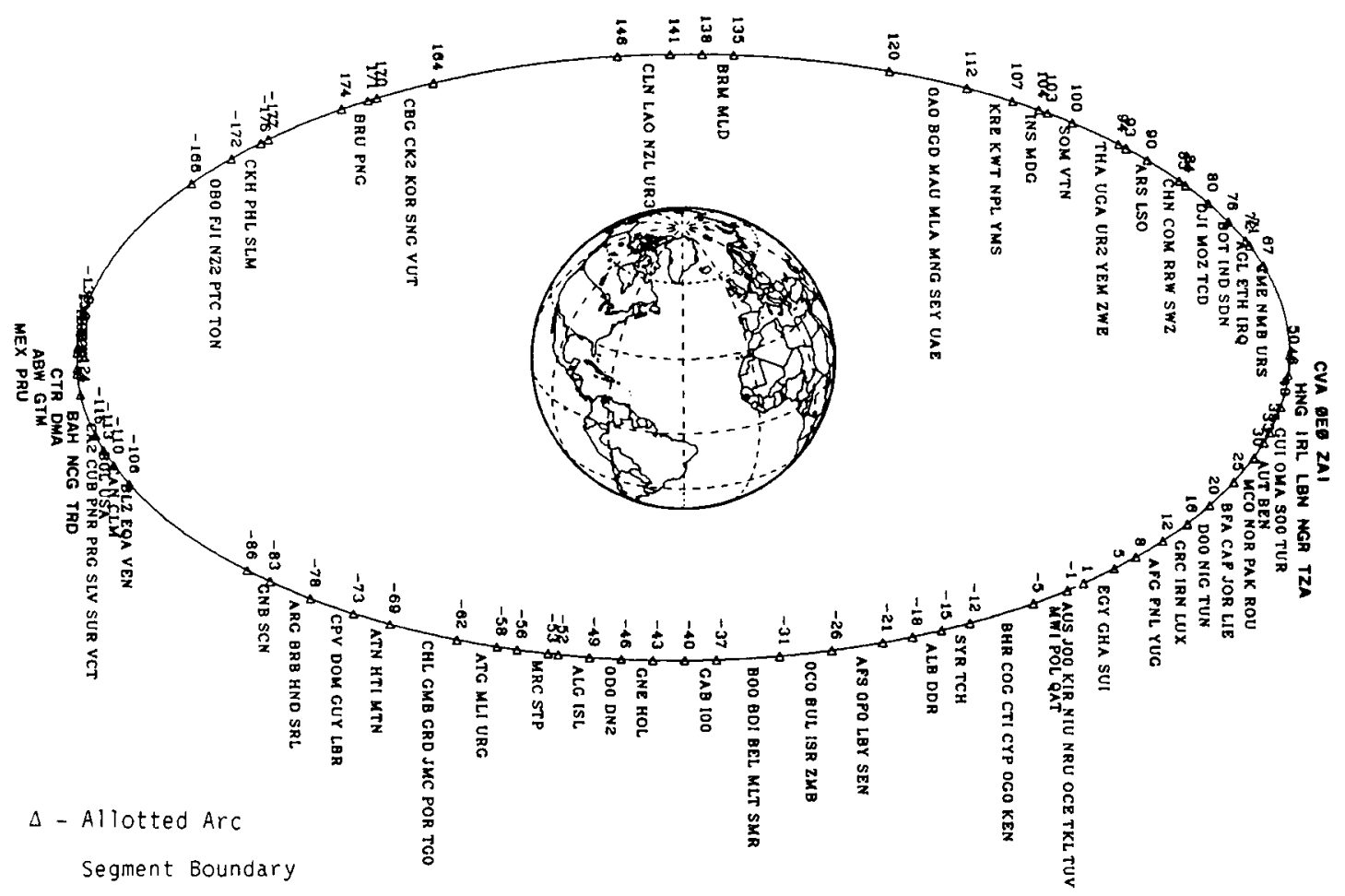

FIGURE 1. - WORLD SCENARIO EXAMPLE (208 SERVICE AREAS - 175 INDIVIDUAL ADMINISTRATIONS, 7 AFFILLIATED SETS).

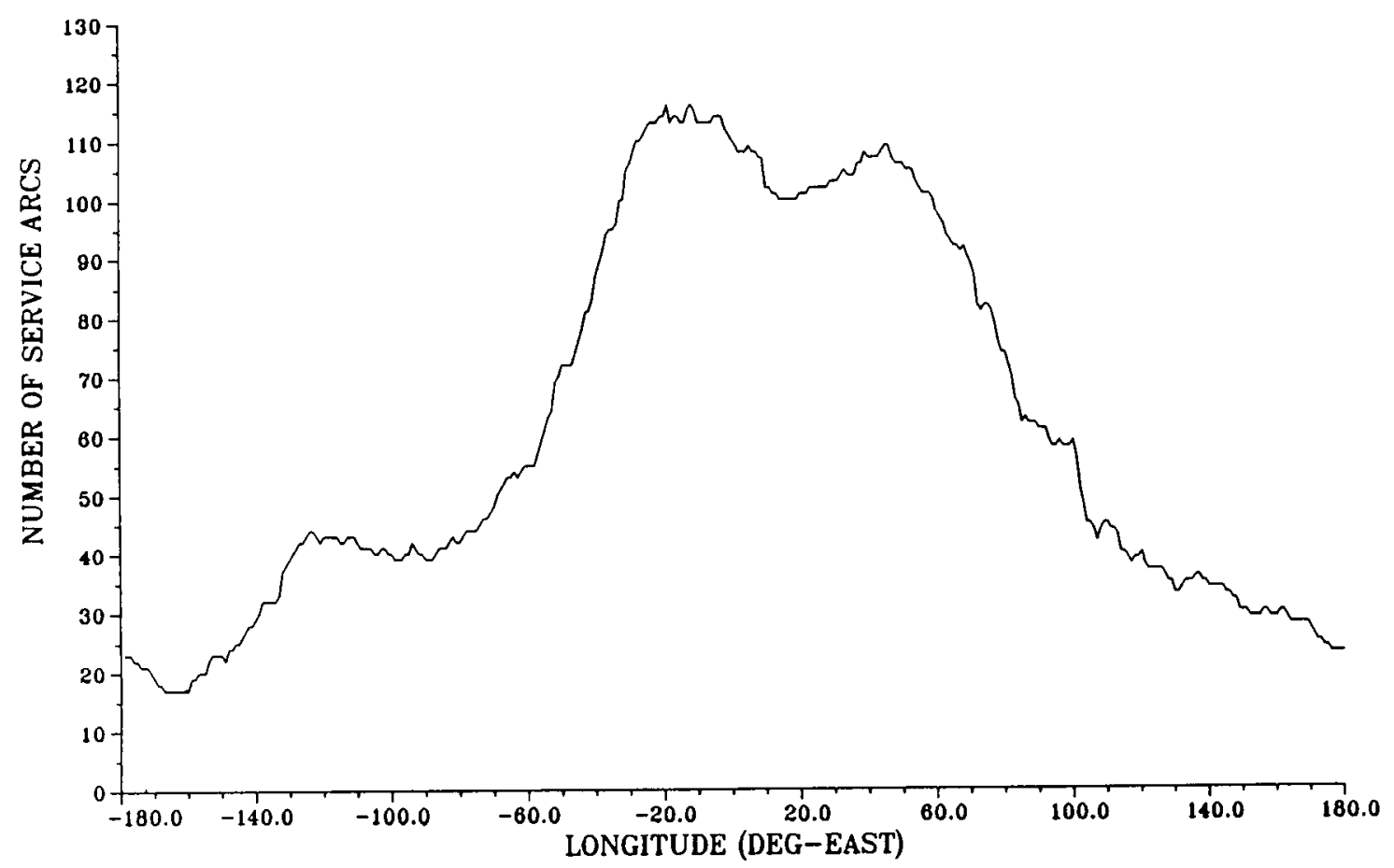

FIGURE 2. - SERVICE ARC DENSITY (WORLD SCENARIO EXAMPLE). 

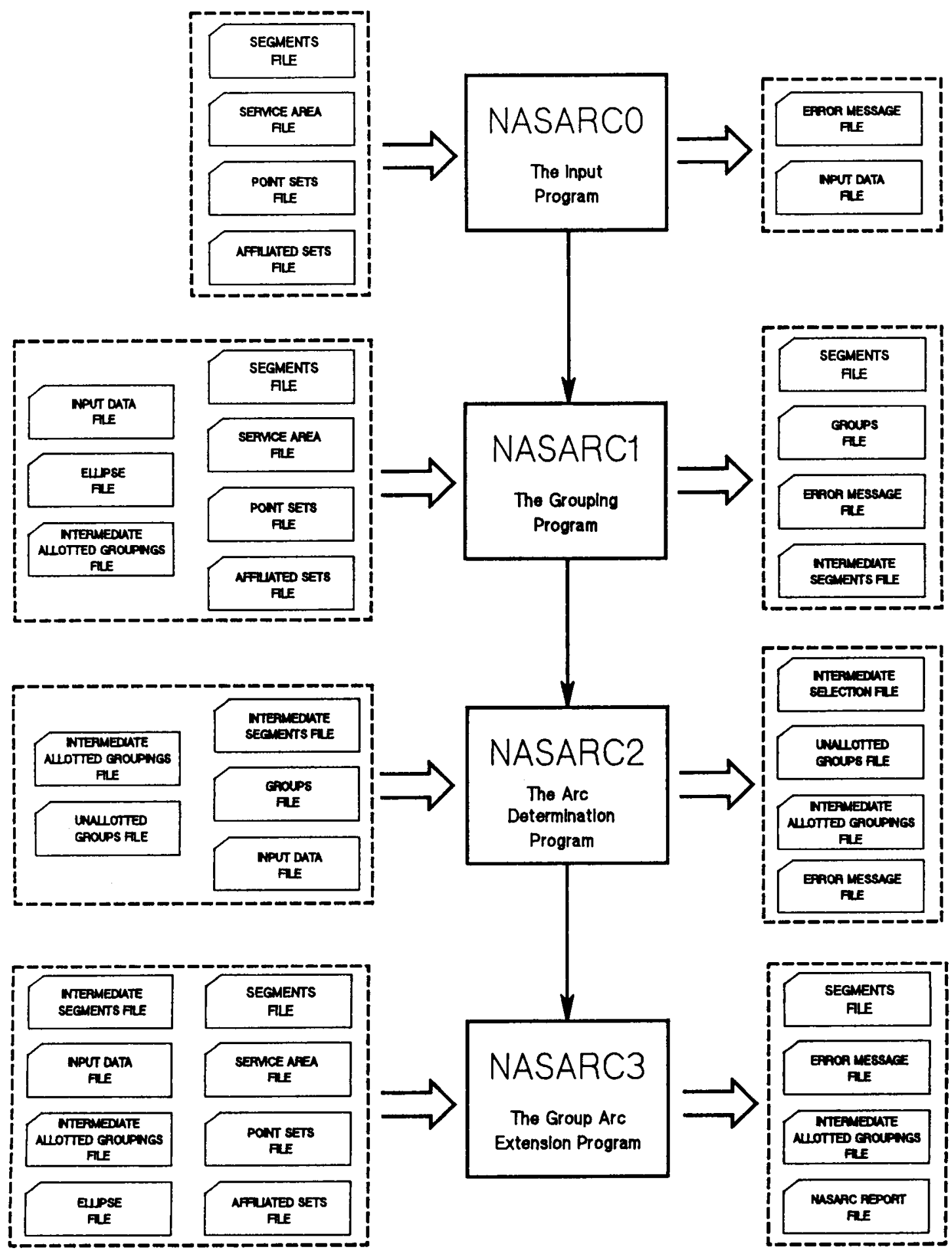

FIGURE 3. - NASARC PROGRAM MODULE FILE OVERVIEW. 


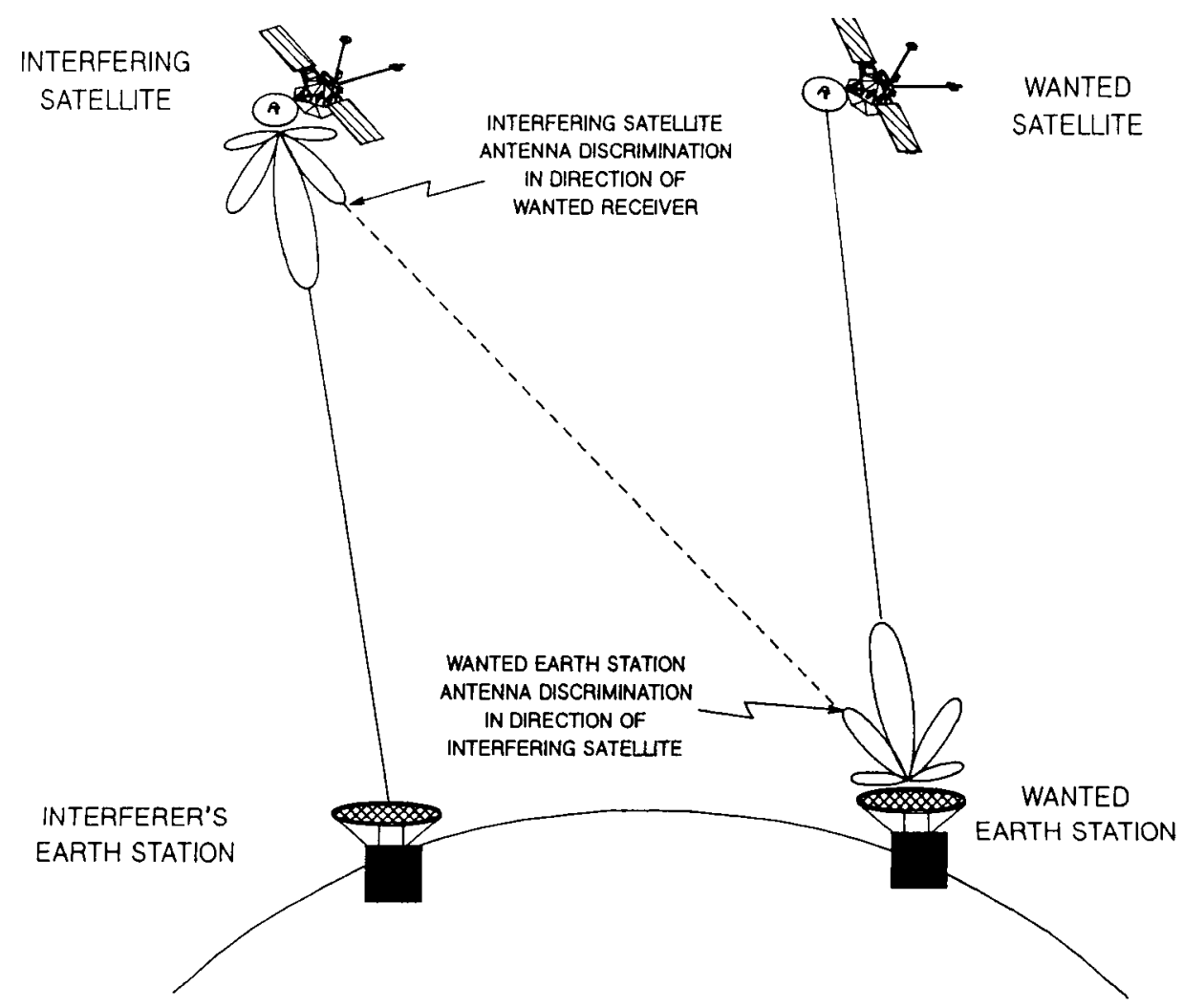

FIGURE 4. - DIAGRAM OF PAIRWISE INTERFERENCE GEOMETRY.
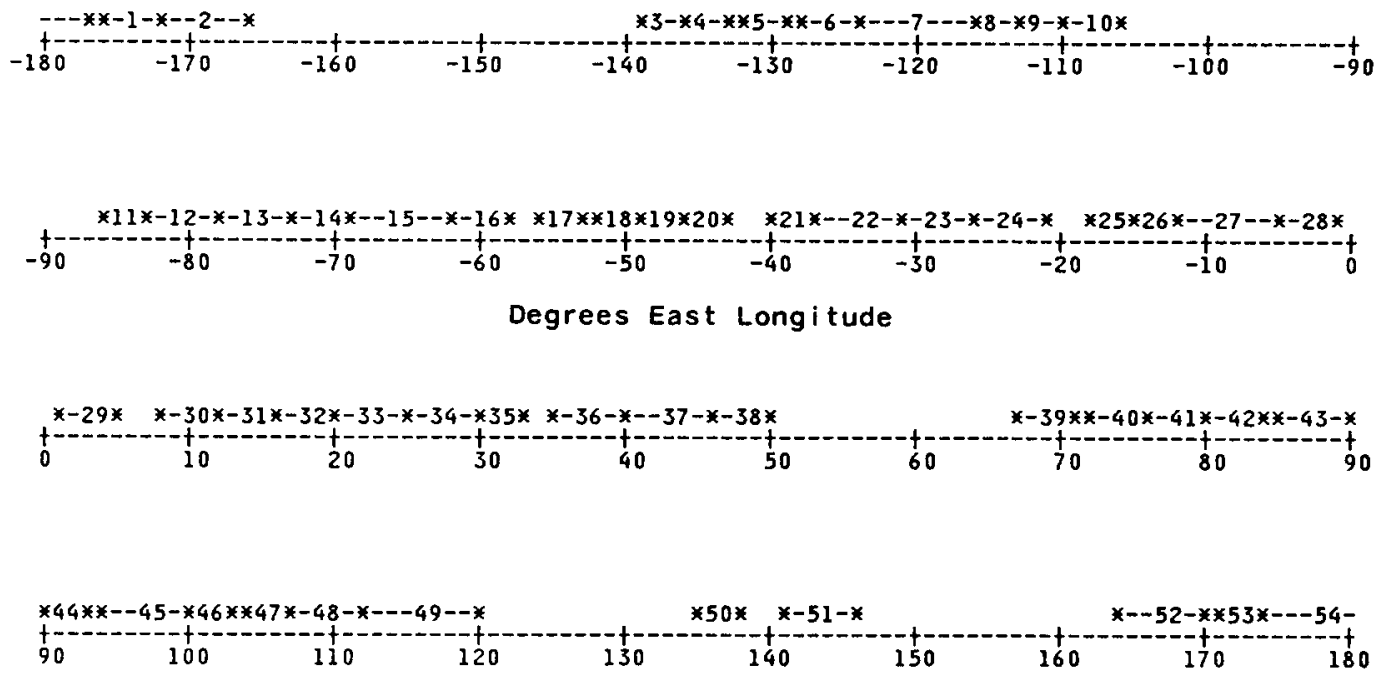

FIGURE 5. - NASARC PDA ORBITAL REPRESENTATION (NUMBERS ON LINE CHART REFER TO GROUPING NUMBERS FOUND IN TABLE 3). 


\begin{tabular}{|c|c|c|c|}
\hline \multicolumn{4}{|c|}{$\bigcup_{\substack{\text { National Aeronautics and } \\
\text { Space Administration }}}$} \\
\hline & $\begin{array}{l}\text { Report No. NASA TM- } 100789 \\
\text { AIAA- } 88-0788\end{array}$ & 2. Government Accession No. & 3. Recipient's Catalog No. \\
\hline \multicolumn{3}{|c|}{ 4. Title and Subtitle } & 5. Report Date \\
\hline \multicolumn{3}{|c|}{$\begin{array}{l}\text { Numerical Arc Segmentation Algorithm for a } \\
\text { Radio Conference - A Software Tool For Communication } \\
\text { Satellite Systems Planning }\end{array}$} & 6. Performing Organization Code \\
\hline \multirow{2}{*}{\multicolumn{3}{|c|}{$\begin{array}{l}\text { 7. Author(s) } \\
\text { W.A. Whyte, A.O. Heyward, D.S. Ponchak, R.L. Spence, } \\
\text { and J.E. Zuzek }\end{array}$}} & $\begin{array}{l}\text { 8. Performing Organization Report No. } \\
\text { E-3962 }\end{array}$ \\
\hline & & & $\begin{array}{l}\text { 10. Work Unit No. } \\
643-10-01\end{array}$ \\
\hline \multirow{3}{*}{\multicolumn{3}{|c|}{$\begin{array}{l}\text { 9. Performing Organization Name and Address } \\
\text { National Aeronautics and Space Administration } \\
\text { Lewis Research Center } \\
\text { Cleveland, Ohio } 44135-3191\end{array}$}} & \\
\hline & & & 11. Contract or Grant No. \\
\hline & & & 13. Type of Report and Period Covered \\
\hline \multicolumn{3}{|c|}{ 12. Sponsoring Agency Name and Address } & Technical Memorandum \\
\hline \multicolumn{3}{|c|}{$\begin{array}{l}\text { National Aeronautics and Space Administration } \\
\text { Washington, D.C. 20546-0001 }\end{array}$} & 14. Sponsoring Agency Code \\
\hline \multicolumn{4}{|c|}{$\begin{array}{l}\text { 15. Supplementary Notes } \\
\text { Prepared for the } 12 \text { th International Communications Satellite Systems Conference, } \\
\text { sponsored by the American Institute of Aeronautics and Astronautics, Arlington, } \\
\text { Virginia, March } 13-17,1988 \text {. }\end{array}$} \\
\hline \multicolumn{4}{|c|}{$\begin{array}{l}\text { 6. Abstract } \\
\text { The software package, Numerical Arc Segmentation Algorithm for a Radio Conference } \\
\text { (NASARC), has been developed to provide a method of generating predetermined arc } \\
\text { segments for use in the development of an allotment planning procedure to be car- } \\
\text { ried out at the } 1988 \text { World Administrative Radio Conference (WARC - 88) on the Use } \\
\text { of the Geostationary - Satellite Orbit and the Planning of Space Services Utiliz- } \\
\text { ing It. Through careful selection of the predetermined arc (PDA) for each admin- } \\
\text { istration, flexibility can be increased in terms of choice of system technical } \\
\text { characteristics and specific orbit location while reducing the need for coordina- } \\
\text { tion among administrations. The NASARC software determines pairwise compatibil- } \\
\text { ity between all possible service areas at discrete arc locations. NASARC then } \\
\text { exhaustively enumerates groups of administrations whose satellites can be closely } \\
\text { located in orbit, and finds the arc segment over which each such compatible group } \\
\text { exists. From the set of all possible compatible groupings, groups and their } \\
\text { associated arc segments are selected using a heuristic procedure such that a PDA } \\
\text { is identified for each administration. This paper discusses the various aspects } \\
\text { of the NASARC (Version 2.0) concept and how the software accomplishes specific } \\
\text { features of allotment planning. }\end{array}$} \\
\hline \multicolumn{2}{|r|}{ 17. Key Words (Suggested by Author(s)) } & \multirow{2}{*}{\multicolumn{2}{|c|}{$\begin{array}{l}\text { 18. Distribution Statement } \\
\text { Unclassified - Unlimited } \\
\text { Subject Category } 17\end{array}$}} \\
\hline & $\begin{array}{l}\text { Satellite communicatio } \\
\text { Software } \\
\text { Allotment planning } \\
\text { Predetermined arcs }\end{array}$ & $\begin{array}{l}\text { Unclassif } \\
\text { Subject } c\end{array}$ & \\
\hline \multicolumn{2}{|r|}{$\begin{array}{l}\text { 19. Security Classif. (of this report) } \\
\text { Unclass if ied }\end{array}$} & $\begin{array}{l}\text { Security Classif. (of this page) } \\
\text { Unclassified }\end{array}$ & $\begin{array}{c}\text { 21. No of pages } \\
16 \\
\end{array}$ \\
\hline
\end{tabular}

